\title{
Enseñar español en primaria: análisis desde el interaccionismo sociodiscursivo
}

\author{
Teaching Spanish in Elementary School: An Analysis from \\ Sociodiscursive Interactionism
}

Blanca Araceli Rodríguez Hernández (1) https://orcid.org/0000-0001-8876-7502

Erika Michelle González López (2) https://orcid.org/0000-0003-0534-4198

(1) Universidad Autónoma de San Luis Potosí

(2) Secretaría de Educación de San Luis Potosí

(Recibido: 6 de marzo de 2019; Aceptado para su publicación: 12 de julio de 2019)

Cómo citar: Rodríguez, B. A. y González, E. M. (2020). Enseñar español en primaria: análisis desde el interaccionismo sociodiscursivo. Revista Electrónica de Investigación Educativa, 23, e03, 1-14. https://doi.ora/10.24320/redie.2021.23.e03.2908

\section{Resumen}

El artículo presenta los resultados del análisis del trabajo de una profesora de primaria durante una clase de español en un grupo de quinto grado. La perspectiva teórica de referencia es el interaccionismo sociodiscursivo y sus contribuciones al estudio del trabajo de los docentes a partir del trabajo prescrito, efectivo y representado. Para el análisis de la clase se retoma la propuesta de Riestra (2008), para estudiar las consignas y el diálogo sobre éstas a través de la metodología de la autocofrontación entre la investigadora y la profesora de grupo. Los resultados muestran la importancia de la reformulación de las consignas para comprender la evolución de las tareas y evaluar lo que sucede en la clase. Asimismo, dejan entrever que los motivos de las reformulaciones estuvieron vinculados tanto con la profesora (supuestos gestión de clase y confusión con el género textual) como con el trabajo prescrito.

Palabras clave: evaluación del docente, estudio del trabajo, asignaturas de enseñanza básica, español.

\section{Abstract}

This article presents the results of an analysis of the work of an elementary school teacher in a fifth-grade Spanish class. The theoretical frame of reference is sociodiscursive interactionism and its contributions to research on teachers' work based on prescribed, effective and represented work. To analyze the class, we revisit Riestra's (2008) proposal to study the instructions given and associated dialogues through selfconfrontation between the researcher and the class teacher. The results show the importance of rephrasing instructions to understand how tasks progress and evaluate what is happening in the classroom. They also suggest that the reasons for rephrasing were linked both to the teacher (class management assumptions and confusion about the genre of the text) and to the prescribed work. 


\section{Introducción}

En el ámbito académico el trabajo de los profesores ha sido objeto de estudio de investigaciones etnográficas que han aportado importantes descripciones sobre realidades escolares o extraescolares no documentadas y, con ello, han contribuido a la comprensión de las prácticas docentes desde sus propios actores y en los contextos en donde se sitúan (Arteaga, 2011; Cano, 2017; Edwards, 1993). Recientemente, investigaciones didácticas disciplinares que antes enfocaron su interés en los alumnos y en los procesos de aprendizaje han tomado conciencia de la necesidad de interesarse también en la enseñanza y en la realidad del trabajo del profesor [Goicoechea et al. (2011), Riestra (2017), Tapia (2017) y Tapia y Goicoechea (2015) en didáctica de las lenguas; Civera (2017) en matemáticas, y Goicoechea y Goicoechea (2017) en educación física].

En conjunto, los resultados de dichas investigaciones muestran que los maestros poseen un amplio repertorio de conocimientos que ponen en juego en sus aulas para alcanzar lo que Bronckart (2007) denomina la especificidad de su labor: "La gestión de una situación de clase y el desarrollo de la lección en función de las expectativas y objetivos de la institución escolar y las características y reacciones de los alumnos" (p. 170). Además, destacan que entender esta especificidad implica visibilizar el trabajo docente a través de identificar las debilidades y fortalezas del profesor in situ.

Por otra parte, en el ámbito de las políticas educativas, a partir de 2014, el Instituto Nacional para la Evaluación de la Educación (INEE) comenzó a evaluar a los profesores para designar su idoneidad en el cargo. La evaluación contempló los conocimientos de los maestros a través de un examen y la autorreflexión sobre su práctica guiada por preguntas llamadas "tareas evaluativas". Esta autorreflexión, a nuestro juicio, complejizó la tarea porque los profesores no contaron con apoyo para sistematizar y analizar la información que recogían. Además, la evaluación en general estuvo mediada por factores que convirtieron el intento del INEE por acercarse al trabajo de los profesores en un requerimiento laboral, más que en una alternativa para mejorar las prácticas docentes.

Lo expuesto, desde los ámbitos académico y político, permite sostener que es necesario seguir analizando el trabajo de los profesores en las aulas para ayudar a comprender la relativa opacidad de su labor y orientar la construcción de formas de evaluación apropiadas a las características de la actividad que desarrollan.

El objetivo de este artículo es doble: por un lado, se presentan los resultados del análisis del trabajo de una profesora de primaria durante una clase de español con el fin de contribuir a la evaluación de la enseñanza que ahí se desarrolló. La pregunta que dirigió el análisis fue: ¿Qué aspectos del trabajo de la profesora pueden orientar la evaluación de la enseñanza? Por otro lado, se comentan algunos aspectos metodológicos considerados relevantes, pues es un primer acercamiento empírico con una perspectiva teórica de fuerte tradición en otros países, pero que no ha sido utiliza en México: el interaccionismo sociodiscursivo (ISD).

El ISD sustenta una concepción de la didáctica de las lenguas como disciplina en construcción (Bronckart, 2013) que se apoya en préstamos teóricos de la lingüística, la psicología, la filosofía y la sociología, pero que mantiene su propio objeto de estudio alrededor de "las complejas relaciones entre los tres polos del triángulo didáctico: profesor, alumno y la lengua o las lenguas enseñadas" (Dolz et al., 2009, p. 118). En este tenor, uno de los objetos de estudio del ISD es el trabajo del profesor, pues su análisis permite entender estas relaciones.

Los resultados que aquí se reportan se inscriben en una investigación que retomó el ISD para evaluar la implementación de una secuencia didáctica orientada a enseñar a escribir artículos de opinión a alumnos de primaria. ${ }^{1}$ El eje orientador de esta evaluación fue el análisis del trabajo docente.

\footnotetext{
1 Proyecto "Secuencias didácticas de lengua basadas en el género textual: piloteo de una metodología didáctica"; financiamiento PRODEP, registro UASLP-PTC-613.
} 
El interés por estudiar el trabajo docente desde los aportes del ISD radica no sólo en la evaluación de la secuencia didáctica si no, y principalmente, en la posibilidad de contribuir con los profesores para que reflexionen acerca del desarrollo de una situación de clase y sobre los obstáculos que enfrentan para, de esa manera, contribuir a mejorar su desempeño.

\subsection{El análisis del trabajo desde el ISD}

En el marco del ISD, Bronckart (2007) refiere a la actividad de enseñanza como un verdadero trabajo, el trabajo del profesor. Como resultado de una amplia investigación, este autor discute la relativa opacidad del trabajo docente y destaca las dificultades para describirlo y caracterizarlo desde el espacio en donde ocurre, el aula, y propone una metodología de análisis basada en desarrollos teórico-prácticos provenientes del campo de la ergonomía a partir de tres dimensiones: trabajo prescrito, trabajo real y trabajo representado.

Goicoechea (2017), -basada en los estudios sobre el trabajo de Clot y Fäita (2000)- discute la pertinencia de hablar de lo real del trabajo versus lo efectivo, y como resultado adecua el dispositivo de Bronckart. La autora propone trabajo prescrito, efectivo y representado, y analiza clases de diferentes profesores argentinos. Esta investigación se suscribe a dicha categorización.

El trabajo prescrito representa lo que debería ser la prescripción y está registrado en los documentos prefigurativos que orientan la actividad del profesor, como el currículum o el plan de clase. Para analizarlo se revisan ambos documentos.

El trabajo efectivo, por su parte, es "la reconfiguración de las acciones en situación de trabajo" (Goicoechea et al., 2011, p. 2); es decir, es lo efectivamente realizado en clase. Para analizarlo se estudian las interacciones entre el profesor y los alumnos.

Por último, el trabajo representado es lo implícito en las acciones del profesor. Para indagar estas representaciones, Bronckart (2007) entrevistó a los trabajadores de su estudio antes y después de la ejecución de las tareas. "Este tipo de análisis tiene por objetivo observar cómo quien enseña concibe su propio actuar" (Bronckart, 2007, p. 176); en el presente trabajo no fue posible contar con la entrevista previa, si bien se contempló la entrevista propuesta por Riestra (2008) para identificar las prefiguraciones del actuar de los profesores, la maestra participante no contó con el tiempo suficiente para concretar la entrevista antes de iniciar la intervención. Por ello se recogieron las concepciones de su actuar docente después de la clase a través de la metodología de la autoconfrontación.

Desde los aportes de Fernández y Clot (2010) y Goicoechea (2017), la autoconfrontación permite al docente enfrentar su propia práctica con ayuda de los investigadores, y reflexionar sobre lo realizado para expresar las intenciones que subyacen a la actividad que lleva a cabo. Este proceso ayuda al investigador a conocer cómo concibe el docente su propio actuar y brinda insumos al profesor para identificar lo que puede mejorar en sus próximas intervenciones.

En el trabajo docente, lo prescrito, efectivo y representado se concretan en lo que sucede en el aula, por lo que esta investigación se centró en las interacciones en clase, enfocándose en la intervención y negociación entre profesora y alumnos, apoyándonos en las dos dimensiones restantes. Para analizar las interacciones, nos centramos en las consignas de clase.

Para Riestra (2008), las consignas son instrumentos mediadores de la enseñanza que funcionan como eslabones dialógicos a través de los que el profesor interactúa mentalmente con los alumnos en sus procesos de aprendizaje. La autora distingue consignas en sentido estricto y en sentido amplio: las primeras refieren a los segmentos de textos que definen e inician la actividad producida en clase; mientras que las segundas son las producciones verbales de los enseñantes que encuadran y comentan las consignas en sentido estricto. 
Las relaciones que se establecen entre las intenciones de los profesores (observadas en las consignas) y las acciones realizadas por los estudiantes (ya sea en sus producciones textuales o en las respuestas orales a las preguntas de los maestros) permiten analizar el trabajo docente.

\section{Método}

Los trabajos que estudian las interacciones en clase pueden enfocarse tanto en la intervención y negociación entre profesor y alumnos como en los efectos que la intervención produce en la tarea que realizan los estudiantes (Riestra, 2008). Este trabajo se centra sólo en la primera posibilidad; se analiza una clase con un grupo de quinto grado de primaria. ${ }^{2}$ Se eligió esta clase por la dificultad que representó analizarla por la cantidad de diálogo sobre las consignas.

El grupo de quinto grado estuvo formado por 24 alumnos de entre 10 y 11 años, de una escuela primaria de organización completa de la periferia de San Luis Potosí (México). La profesora tiene siete años de servicio; cursó una licenciatura y una maestría, ambas en educación primaria, en una escuela normal.

Para analizar las interacciones entre la profesora y los estudiantes a través de las consignas, las clases se grabaron en audio y se transcribieron. ${ }^{3}$ La grabación de la clase analizada fue de 55 minutos.

El análisis se desarrolló en dos fases. En la fase 1 se abordó el trabajo efectivo. Primero, se revisó la planeación contenida en la secuencia didáctica (el trabajo prescrito); a partir de ésta se recuperó:

a. el objetivo de enseñanza: lo que se espera que los alumnos aprendan,

b. los momentos de clase: la organización de las actividades según estuvieran orientadas a leer, discutir oralmente o escribir, y

c. las indicaciones establecidas en el diseño didáctico a las que llamamos consignas prescritas para diferenciarlas de aquellas que la profesora usó durante la implementación.

Después se leyó la transcripción para identificar las consignas en sentido estricto y en sentido amplio (Riestra, 2008). En total, se identificaron y analizaron cuatro conjuntos de producciones:

- 3 consignas prescritas

- 5 consignas en sentido estricto

- 15 consignas en sentido amplio

- Reacciones orales de los alumnos a las consignas

Para analizar los tres grupos de consignas se identificaron las tareas y las nociones propuestas en cada una de ellas. Se entiende "tarea" como la finalidad que persigue la consigna. Las nociones son los objetos de saber vehiculizados en las consignas (Riestra, 2008) y, por lo tanto, perseguidos en clase. Pueden ser nociones teóricas cuando se trata de términos técnicos o didácticas cuando son términos técnicos didactizados.

Con esta información se elaboró una tabla de tres columnas; la primera para las consignas prescritas, la segunda para las de sentido estricto y la tercera las de sentido amplio, todas alineadas en la misma fila. En la fila se marcaron las tareas, nociones teóricas y didácticas contenidas en cada consigna. Cada consigna se marcó con un código: CP seguido de número para las consignas prescritas, CSE seguido de número para las consignas en sentido estricto y este mismo código más _A seguido de número para las reformulaciones. Esto permite mantener la referencia al trabajo prescrito àl observar las consignas en sentido estricto y sus reformulaciones (tabla 1 ).

\footnotetext{
${ }^{2}$ La secuencia didáctica se implementó en 11 sesiones en octubre y noviembre de 2018.

${ }^{3}$ La escuela no permitió que las clases se grabaran en video, por lo que únicamente se registró el audio.
} 
Tabla 1. Análisis de tareas, nociones teóricas y didácticas de cada consigna

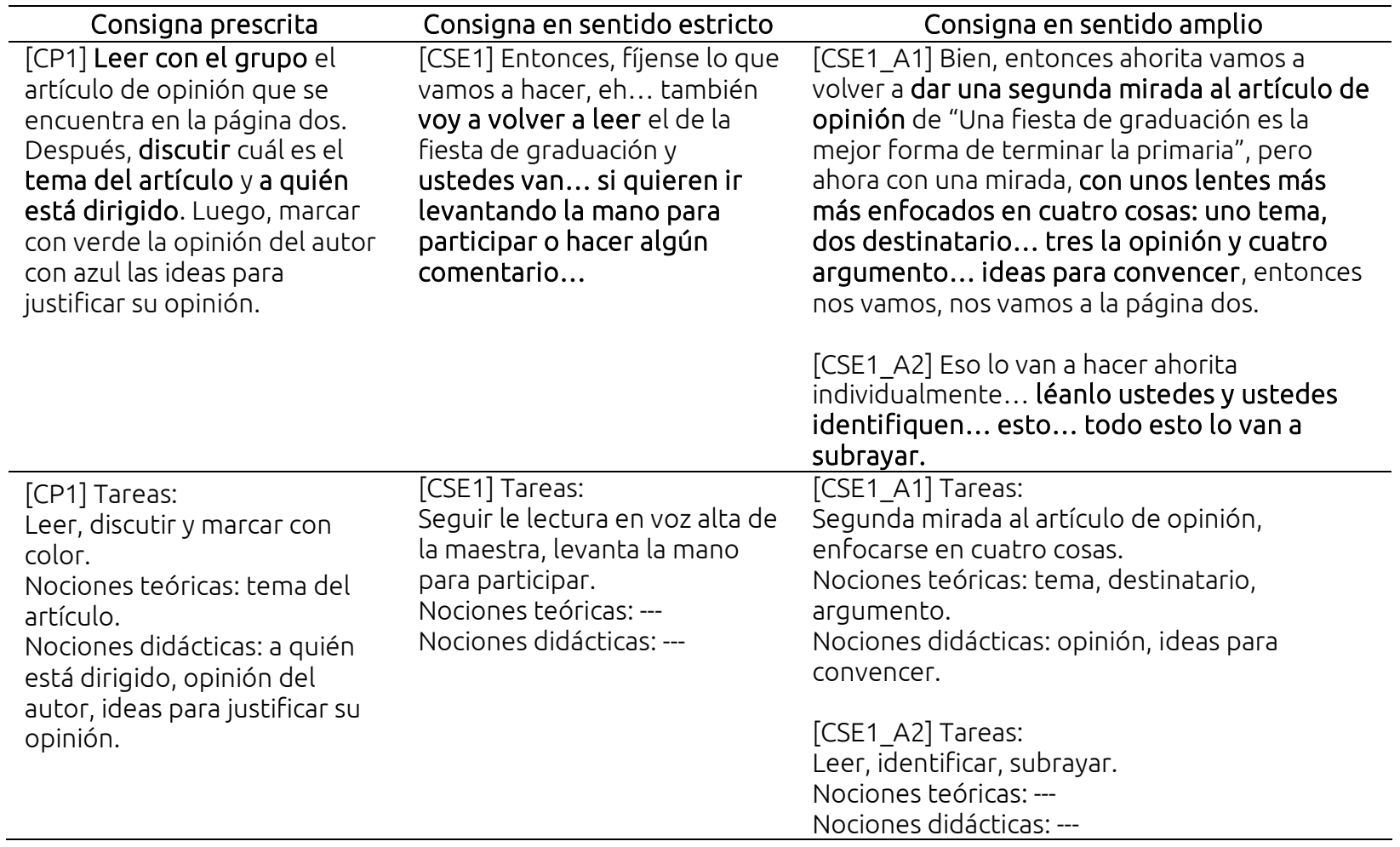

La fase 2 se abocó a la autoconfrontación entre profesora e investigadora. Primero, se revisó el objetivo de enseñanza de la sesión y las consignas del trabajo prescrito; luego, se leyó la transcripción de la clase; después la profesora comentó lo que considerara relevante y por último, se presentó la tabla producto de la fase 1 para discutirla y reorganizarla a partir de los sentidos que la profesora atribuía a su trabajo.

La autoconfrontación duró 70 minutos, se grabó en audio y se transcribió. Cabe puntualizar que la profesora es coautora de este trabajo, por lo que su punto de vista está integrado en el análisis.

\section{Resultados}

La clase tuvo como objetivo de enseñanza que los alumnos leyeran y analizaran un artículo de opinión para identificar el tema, la opinión, los argumentos y el destinatario. Dado que se trataba del primer acercamiento de los alumnos con este género textual, se diseñaron actividades sobre un texto modelo.

La clase se organizó en tres momentos, que cambiaron durante la intervención, pues se modificó tanto la tarea solicitada (leer, discutir o escribir) como la forma de organización prescrita (individual, en equipos o grupal), como se ve en la tabla 2.

Tabla 2. Momentos de organización de la clase y su concreción en la intervención

\begin{tabular}{l|l}
\hline \multicolumn{1}{c|}{ Momentos planeados en el diseño } & \multicolumn{1}{c}{ Momentos ocurridos en la implementación } \\
\hline Actividad de lectura grupal sobre el texto modelo: & Actividad de lectura, discusión y trabajo sobre el \\
identificar tema, destinatario, opinión y argumentos. & texto modelo (individual-equipos). \\
Actividad de discusión en parejas para comentar la & Actividad de discusión y escritura (grupal-individual). \\
intención del autor en el texto modelo. & \\
Actividad de escritura individual. & \\
\hline
\end{tabular}


Рara presentar lo que sucedió en los dos momentos de clase se analizaron tres consignas en sentido estricto: una actividad de lectura (inicio de la clase), una de escritura (a mitad de la clase) y una de evaluación (cierre).

\subsection{Actividad de lectura}

La tabla 3 muestra el desarrollo de la actividad de lectura desde la planeación hasta la última consigna ampliada.

Tabla 3. Consignas de lectura

\begin{tabular}{|c|c|c|}
\hline Consigna prescrita & Consigna en sentido estricto & Consigna en sentido amplio \\
\hline $\begin{array}{l}\text { [CP1] Leer con el grupo } \\
\text { el artículo de opinión que } \\
\text { se encuentra en la página } \\
\text { dos. Después, discutir } \\
\text { ¿cuál es el tema del } \\
\text { artículo? y ¿a quién está } \\
\text { dirigido? Luego, marcar } \\
\text { con verde la opinión del } \\
\text { autor y con azul las ideas } \\
\text { para justificar su } \\
\text { opinión. }\end{array}$ & $\begin{array}{l}\text { [CSE1] Entonces, fíjense lo que } \\
\text { vamos hacer, eh... también voy } \\
\text { a volver a leer el de la fiesta de } \\
\text { graduación y ustedes van... si } \\
\text { quieren ir levantando la mano } \\
\text { para participar o hacer algún } \\
\text { comentario... }\end{array}$ & $\begin{array}{l}\text { [CSE1_A1] Bien, entonces ahorita vamos a volver a } \\
\text { dar una segunda mirada al artículo de opinión de } \\
\text { "Una fiesta de graduación es la mejor forma de } \\
\text { terminar la primaria", pero ahora con una mirada, } \\
\text { con unos lentes mas más enfocados en cuatro } \\
\text { cosas: uno tema, dos destinatario... tres la } \\
\text { opinión y cuatro argumento... ideas para } \\
\text { convencer, entonces nos vamos, nos vamos a la } \\
\text { página dos. } \\
\text { [CSE1_A2] Eso lo van hacer ahorita } \\
\text { individualmente... léanlo ustedes y ustedes } \\
\text { identifiquen... esto... todo esto lo van a } \\
\text { subrayar. }\end{array}$ \\
\hline
\end{tabular}

La consigna prescrita (CP1) planteaba tres tareas: leer, discutir y trabajar sobre el texto modelo, todas ellas de forma grupal. La discusión se propuso sobre una noción teórica (tema) y una noción didáctica (a quién está dirigido el texto); mientras que el trabajo sobre el artículo implicaba marcar dos nociones didácticas (opinión e ideas para justificar). Como puede observarse, una noción teórica no está didactizada en la consigna.

En la implementación, la consigna en sentido estricto (CSE1) planteó dos tareas grupales: leer y comentar en voz alta. Después, la profesora la reformuló (CSE1_A1): agregó información sobre el trabajo con el texto modelo, es decir, indicó en qué aspectos se iban a concentrar. Finalmente, hubo una nueva reformulación (CSE1_A1): cambió la organización de la actividad, pasó de una organización grupal que implicaba el trabajo guiado a una lectura individual en la que, al mismo tiempo, los alumnos debían trabajar sobre el texto e indicó que debía subrayarse todo (tema, destinatario, opinión y argumentos), en lugar de únicamente la opinión y los argumentos.

Las dos consignas ampliadas muestran que la profesora fue reformulando la consigna en sentido estricto para recuperar la indicación planificada, que finalmente no logró comunicar a los niños.

A partir de la autoconfrontación, se identificó que durante la clase la profesora no se percató de las reformulaciones que realizó y se sentía insegura porque no revisó a profundidad su planeación, como se evidencia en el siguiente fragmento de la entrevista:

M: Hasta ahorita que lo veo lo noto... había pensado mucho, lo había planificado mucho como la lectura y la puesta en situación, pero... ya al momento de... o sea, no estuvo tan planificado, desde mi... ahorita desde mi punto de vista... pienso yo que por eso estaba insegura.

E: ¿Qué te daba inseguridad? 
M: Sí, o sea, como... un día antes la chequé (la planeación), dije bueno... o sea, nada más la leí, o sea, dije nada más leerla y ya, pero ya en el momento (de la clase) no, dije... pienso yo que fue esa la inseguridad de hacer tantas intervenciones. (Autc3_Frag1). ${ }^{4}$

Por otra parte, a partir las reacciones orales de los alumnos a la última consigna ampliada se identificó que enfrentaron dificultades para concretarla. Una de las complicaciones que enfrentaron los estudiantes fue para encontrar el tema del artículo de opinión y subrayarlo. El tema, en tanto asunto o materia de un discurso, algunas veces puede mencionarse y hacerse explícito, pero otras no. En cualquier caso, nunca compete sólo a una parte en específico, sino al texto completo. En el artículo que trabajaron los niños el tema debía inferirse a partir de la lectura.

En la clase, la profesora usó una noción didáctica (¿de qué habla?) para referirse al tema. En el fragmento I puede observarse que la primera intervención de la maestra no hizo eco, pues el estudiante intentó buscar en su texto algo para subrayar y cumplir así con la tarea encomendada, y la segunda no se respondió.

M: A ver, el tema, ¿de cuál es el gran tema de este artículo?

N2: Esto (el alumno señala un fragmento en el texto).

M: No, el gran tema, ¿de qué habla esto (el artículo)? (Clase3_Fragl). ${ }^{5}$

En el fragmento II la maestra nuevamente utiliza una noción didáctica para reformular la noción teórica. Ante esto, uno de los alumnos responde de forma adecuada, aunque la profesora continúa hasta que, finalmente, termina dándoles una respuesta.

M: A ver, el tema ¿de cuál es el gran tema de este artículo?

N40: Esto (señala el escrito).

M: No, el gran tema ¿de qué habla esto?

N40: Ah... de que los niños quieren hacer una fiesta para festejar el último día, que hacen.

M: Ajá, entonces, ¿cuál es el tema? Mira, por ejemplo, la vez pasada dimos ideas ¿sobre?... Las tareas, si son buenas o malas hacer las tareas... ¿Cuál sería el gran tema de todo este artículo?

N41: Pos, que estamos de acuerdo que hagan fiestas.

M: ¿De la fiesta de...? ¿Fiesta nada más?

N42: Graduación.

M: Ah, el tema sería fiesta de graduación. (Clase3_Fragll).

La consigna en sentido amplio también requería que los alumnos identificaran el destinatario del texto y lo subrayaran. En algunos géneros textuales, como por ejemplo la carta, el destinatario está explícito y puede encontrarse con relativa facilidad en el texto; sin embargo, en el artículo de opinión el destinatario no aparece de manera explícita. Para identificar a qué audiencia se dirige y a quién se espera convencer es necesario leer el texto e inferirlo.

\footnotetext{
4 Simbología: M señala la voz de la Maestra y E la voz de la Entrevistadora.

${ }^{5}$ Simbología de transcripción: M marca el turno de la maestra; N seguido de número indica la participación de los niños; se utilizan paréntesis para insertar notas aclaratorias y en fragmentos posteriores se emplean puntos suspensivos para marcar pausas.
} 
A partir de esta indicación, los niños esperaban encontrar escrito el nombre del destinatario, por lo que algunos lo atribuyeron al autor y otros inferían la audiencia del texto, pero no podían subrayarla.

En el fragmento III se muestra la interacción entre la profesora y un alumno que subrayó el autor. A partir de la intervención de la maestra puede suponerse que el estudiante sabe que se trata del autor y no del destinatario, pero aun así subrayó. La profesora intentó ayudarlo, pero seguía esperando que subrayara.

M: ¿Ya identificaste a quien va dirigido? ¿Ese será a quién va dirigido o quién es él? (Se refiere al nombre del autor).

N36: Eh... el que lo escribió.

M: Entonces, ¿para él va dirigido? No, ¿verdad? ¿Cómo se le llama al que lo escribió?

N36: José Antonio Torres Pérez.

M: Ajá, pero él es el autor ¿Sí? Entonces, tú vas a buscar para quién va dirigido, a quién, a quién quiere convencer ¿Sí? Ese es el destinatario, ¿ya?... ¿Ya subrayaste? (Clase3_FragllI).

En el fragmento IV los niños respondieron correctamente la pregunta de la profesora, es decir, identificaron la audiencia del texto, pero no la subrayaron porque no estaba explícita.

M: ¿A quién va dirigido?

Ns: A los maestros y directores.

M: ¿Dónde dice?...

N45: No viene

M: Y qué argu... ¿No viene? ¿En ningún lado viene a quién quiere convencer? (Clase3_FragIV).

Durante la autoconfrontación, la profesora dijo que estaba pensando en la carta de opinión en donde, a diferencia del artículo de opinión, el destinatario está explícito:

En sexto hay una lección con la carta de opinión, yo estaba pensando en esa carta... desde la primera sesión, eh... de la secuencia... incluso vimos en la transcripción que les dije (a los niños) carta de opinión en lugar de artículo... al final lo dije. No me di cuenta de lo que les pedí, a lo mejor porque pensaba en la carta... quería que encontraran el destinatario (Autc3_Frag2).

Otro de los aspectos que los niños debían identificar en el texto eran las ideas del autor para convencer; es decir, los argumentos. A diferencia del tema y el destinatario, que pueden ubicarse a partir de inferencias, para encontrar los argumentos es indispensable trabajar sobre el escrito. De otro modo, resulta difícil delimitarlos e identificar los recursos lingüísticos que emplea para escribirlos.

En la consigna prescrita aparece la noción didáctica ideas para convencer, sin embargo, en la implementación, la maestra usó indistintamente la noción didáctica y la noción teórica (argumentos). Los niños no preguntaron en ningún momento el significado de argumentos, pero tuvieron dificultades para identificarlos en el texto.

En el fragmento $V$ se presenta un diálogo entre la profesora y un equipo de estudiantes. Ella retomó la noción didáctica para referirse a los argumentos, a partir de esto, uno de los alumnos decidió consultar el texto modelo y otro, inventárselo. 
M: Por eso, o sea, tú estás diciendo que su opinión es que está a favor de la fiesta de graduación, ¿verdad?, entonces, ¿qué ideas da para convencer de que sí se haga la fiesta de graduación?

N141: Ah... entons tengo que checarlo... y aquí (señala el texto) dice qué voy a poner.

M: Exacto.

N142: Yo voy a inventar.

M: No, no, es de lo que leíste, incluso tú ya subrayaste algunas cosas (Clase3_FragV).

En la autoconfrontación la profesora recordó que en la planeación utilizó ideas para convencer como sinónimo de argumentos, porque al tratarse del primer acercamiento con este género textual, sería complicado para los alumnos; sin embargo, lo incorporó durante el desarrollo de la clase.

Creo que fue durante la marcha, o sea... no, no planee decirles es un argumento. Sí habíamos platicado de eso en el diseño de que estaba, pero era muy temprano y se cambió... Pero luego ya en el momento como... consideré que tal vez empezar con palabras clave... Sí, como para explorar y como que fueran ciertas pistas que al primer momento obviamente no iban a llamar la atención, pero ya después... jah, la maestra en la primera situación!... yo pensando que tal vez así un niño pensara ¿no?; jla maestra comentó algo de los argumentos! Y se acordaran. (Autc3_Frag3).

La consigna prescrita no contenía todas las nociones didácticas para todas las nociones teóricas que se abordaban, lo que dificultó el trabajo de la profesora. Además, las tareas con el texto modelo implicaban tanto la lectura guiada como la discusión y el trabajo colectivo. En la implementación no quedaron claras las tareas sobre el texto y se realizaron de forma individual, lo que obligó a la profesora a resolver las dudas de los alumnos en pequeños grupos.

\subsection{Actividad de escritura}

La tabla 4 muestra el desarrollo de la actividad de escritura.

Tabla 4. Consignas de escritura

\begin{tabular}{lll}
\hline \multicolumn{1}{c}{ Consigna prescrita } & \multicolumn{1}{c}{ Consigna en sentido estricto } & \multicolumn{1}{c}{ Consigna en sentido amplio } \\
\hline [CP3] Pedir a los alumnos & [CSE4] El tema del artículo de & [CSE4_A1] Shh individual, no lo estamos haciendo \\
que llenen de forma & opinión una fiesta de graduación & colectivo (los niños responden). No, no, \\
individual la tabla. & es la mejor forma... en esta & individual... shh silencio, tú vas poner si está a \\
& tablita dice, el tema del artículo & favor o en contra de qué (el niño responde). No, \\
& de opinión una fiesta de & es individual, repito la primera: el tema del \\
& graduación es la mejor forma de & artículo de opinión una fiesta de graduación es la \\
& terminar la primaria es... ¿Qué & mejor forma de terminar la primaria es... ¿Cuál es \\
& tema es? (un niño responde) No & el gran tema? ¿De qué habla todo ese artículo? \\
& me la digas a mí, tú escríbela, & (Otro niño responde). \\
& escríbelo. ¿Cuál es el tema el \\
& gran tema del artículo? (el niño \\
& & \\
& &
\end{tabular}

La tarea planteada en la consigna prescrita (CP3) fue llenar individualmente una tabla con cuatro preguntas. Durante la implementación, la consigna en sentido estricto (CSE4) no tiene una tarea explícita; es decir, se alude a la tabla, pero no se dice qué deben hacer los estudiantes. La profesora comienza a leer el contenido de la tabla, hace dos preguntas y, al mismo tiempo, indica que no respondan oralmente, si no por escrito. Más adelante, en la consigna en sentido amplio (CSE4_A1) agrega una tarea explícita "poner", que podría interpretarse como "escribir", pero mantiene la lógica de la consigna en sentido estricto: leer en voz alta las preguntas y aludir a la forma de organización de la actividad. 
Después se desarrolló un extenso diálogo con los alumnos, en el cual identificamos siete reformulaciones más. La maestra orientó la actividad leyendo en voz alta las indicaciones de la tabla, pero en ningún momento especificó que debían responder las preguntas por escrito, por lo que continuaron con la misma dinámica.

Durante la autoconfrontación la profesora mencionó que a diferencia de la consigna de lectura, en donde no se percató de las reformulaciones, aquí fue intencional. Decidió llevar la actividad con todo el grupo, para asegurarse que los alumnos comprendieran (ver Autc3_Frag4). Sin embargo, las respuestas orales de los alumnos mostraron confusión, porque interpretaron la lectura en voz alta como una indicación para responder oralmente.

También lo que estoy viendo es eh... sí les doy muchos ejemplos y al final como que también suelo volver a preguntar como que... como que en mi parte de pensar como maestra es como... antes de que escriban algo fijarme si entendieron o no la... no sé si decirlo, la consigna o... o lo que estoy explicando, entonces por eso suelo dar muchas explicaciones y hacer muchos cuestionamientos para cerciorarme si lo están comprendiendo antes de que escriban. (Autc3_Frag4).

\subsection{Actividad de evaluación}

La Tabla 5 muestra el desarrollo de la actividad de evaluación. La consigna en sentido estricto no surgió a partir de la planeación de esta clase. La profesora retomó un ejercicio de evaluación propuesto en la clase anterior para cerrar la sesión.

Tabla 5. Consignas de evaluación

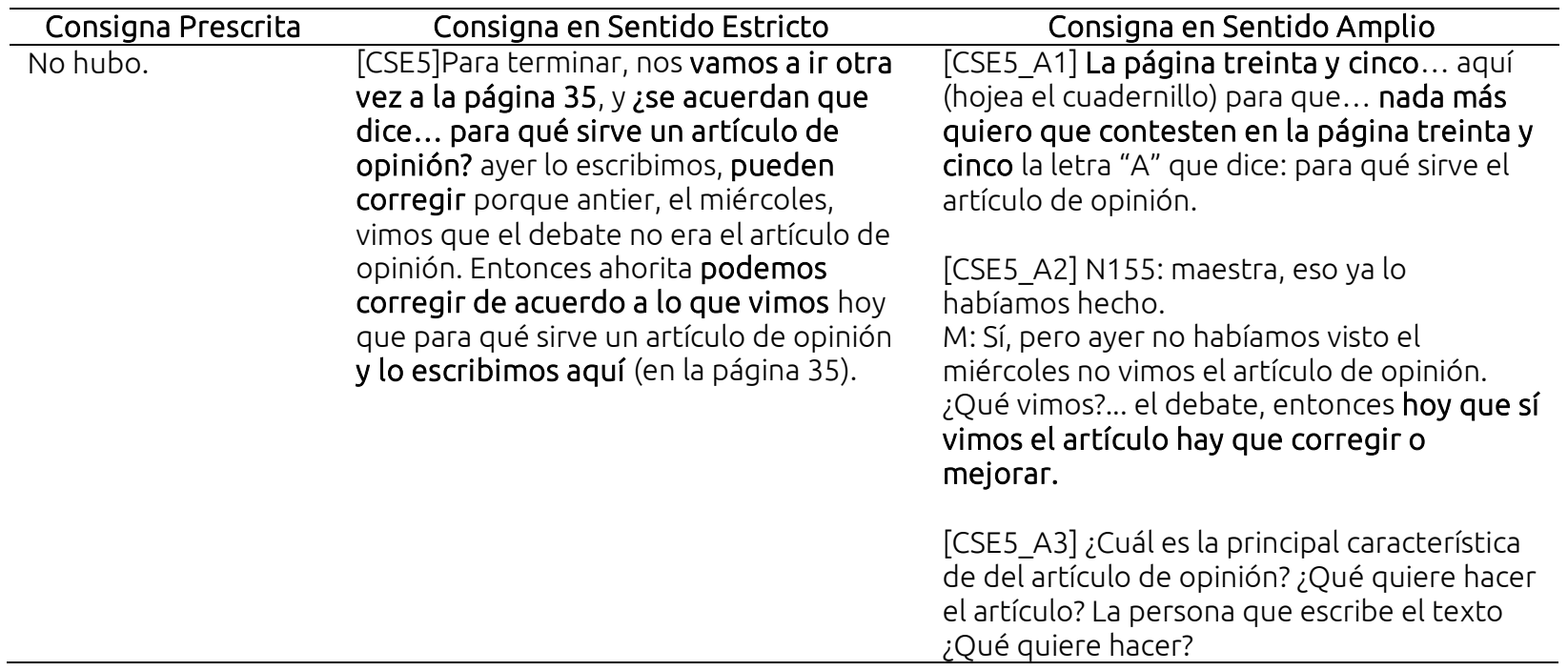

La consigna en sentido estricto (CSE5) planteó tareas explícitas: ir a la página 35, recordar lo que la profesora indicó en la pregunta y corregir lo que habían escrito. Al mismo tiempo estaban implicadas tareas no explícitas como leer lo registrado en la tabla y revisarlo en el marco de lo aprendido en clase.

En la primera consigna ampliada (CSE5_A1) se reformuló para situar la tarea sólo en el inciso A (¿para qué sirve un artículo de opinión?) y no en todos los ejercicios. En la segunda consigna ampliada (CSE5_A2), la profesora aclaró la tarea a partir de la intervención de un alumno. Finalmente, ante la inactividad de los niños (CSE5_A3), la maestra reformuló nuevamente para discutir con el grupo preguntas vinculadas con el inciso $A$. 
En el fragmento VI se muestra la interacción que surgió a partir de esa intervención:

N164: Lo que hicimos aquí atrás.

M: No... un artículo de opinión ¿Qué quiere hacer?

N164: Uy no sé.

M: No, pero él quiere lograr... bueno, así déjalo ¿Tú ya?...

N165: Presente opiniones.

M: Pero, ¿nada más es presentar las opiniones? ¿Qué quiere hacer al presentar la opinión?... Lo principal ¿Qué será? Si tú, por ejemplo, das la opinión de algo y se lo das a alguien, tú, ¿qué quieres hacer para que lo lean?

N164: Ponerle indicaciones.

M: ¿Indicaciones? ¿Es un instructivo? ¿Qué quieres hacer si por ejemplo tú dices quiero...

N165: Una cosa.

M: No, no, a ver, el niño que estaba escribiendo el... el artículo de opinión. ¿Qué quería hacer escribiendo el artículo de opinión?

N166: Hacer una fiesta.

M: Pero, ¿para qué? O sea, sí, pero ¿a quién se lo está escribiendo?, a los maestros, tú me dices que los maestros no querían y él al escribirlo, ¿qué quería hacer?

N164: Convencer a los maestros. (Clase3_FragVI).

En la autoconfrontación la profesora explicó que en la planeación no había una actividad de evaluación, por lo que decidió retomar una de otra clase. Cuando se le preguntó por qué reformuló la tarea inicial para platear preguntas dirigidas a todo el grupo, la profesora respondió:

Porque pensé que en la anterior sesión había quedado como débil... por la premura... ya no se le dio tanta importancia de precisamente hablar sobre lo que ellos entendían... [Sobre] qué era el artículo de opinión y el propósito comunicativo. Si recuerdas, la sesión pasada nada más lo dejo como en una pregunta y lo doy por... como por visto que sí comprendieron y la retomo aquí como pendiente. (Autc3_Frag4).

Ahora bien, a partir de las interacciones se observa que las participaciones diferidas de un reducido grupo de alumnos y orientadas por la intervención de la maestra le dieron pauta para valorar si los niños habían comprendido el propósito comunicativo de un artículo de opinión.

\section{Discusión y conclusiones}

En este artículo presentamos el análisis del trabajo de una profesora de primaria en una clase de español. Los objetivos fueron: contribuir a la evaluación de la enseñanza que desarrolló la maestra y exponer aspectos metodológicos sobre el análisis del trabajo docente.

Con respecto al primer objetivo, el análisis muestra la importancia de la reformulación de las consignas para comprender la evolución de las tareas y evaluar lo que sucede en clase. En Riestra (2008), las reformulaciones orales cumplen la función de andamiaje para guiar las acciones de los alumnos. En esta 
clase, las reformulaciones cambiaron la tarea, introdujeron nociones didácticas que no estaban consideradas en la consigna prescrita y transformaron la forma de organización de la actividad. Los efectos de estas reformulaciones en las reacciones orales de los estudiantes provocaron en algunos momentos confusión y en otros sirvieron de ayuda para concretar la actividad.

Las reformulaciones estuvieron motivadas por sentimientos de inseguridad frente al trabajo prescrito, confusión sobre el género textual que se estaba trabajando y supuestos sobre la gestión de clase. En cuanto a los dos primeros, el trabajo prescrito fue diseñado con la profesora; no obstante, no fue suficiente para aproximarla al conocimiento del objeto de enseñanza y brindarle confianza frente a lo prescrito. En México la enseñanza en la escuela primaria está regida por libros de texto gratuitos, que marcan las actividades a realizar durante el ciclo escolar. Al respecto, investigaciones etnográficas han estudiado las transformaciones que sufre lo prescrito al adaptarse a las eventualidades de la práctica educativa dando pie tanto al currículo real o vivido (Guzmán, 2018) como a diferentes consecuencias emocionales y profesionales en la práctica docente (López, 2007).

En cuanto a los supuestos sobre la gestión de clase que la llevan a implementar acciones para asegurarse de que los estudiantes comprenden la instrucción y aprenden no son exclusivos de esta profesora. A partir de los resultados de investigaciones etnográficas es posible identificar que esto también es relevante para otros maestros.

El supuesto de la profesora que relaciona las reformulaciones de la consigna con la mejor comprensión se identificó en los maestros observados por Edwards (1993). Estos profesores hacían permanentes intervenciones en el transcurso de la lección, no dejaban nada al azar, repetían y señalaban varias veces lo que querían que se aprendiera. En esa misma investigación, la autora observó la dinámica preguntarespuesta como una acción recurrente en las prácticas de los maestros.

A partir de la autoconfrontación, la profesora reflexionó sobre sus decisiones y los motivos que las orientaron, las verbalizó y las relacionó con las reacciones de sus alumnos observadas en el análisis del trabajo efectivo. Esta toma de conciencia de la profesora como profesional, implicó trabajo en conjunto desde la elaboración del plan de clase, el acompañamiento durante la implementación y el análisis de la enseñanza (en este artículo se da cuenta sólo del último momento).

En cuanto a los aspectos metodológicos utilizados, el procedimiento analítico mostró una ruta para estudiar el trabajo docente y evaluar la enseñanza que ameritó modificaciones técnicas a la metodología de referencia para adaptarlo a las condiciones de la escuela, el aula y la profesora. En ese mismo sentido, la prospectiva de este trabajo es, en primer término, hacia la propia investigación. Al tratarse de una investigación empírica basada en una perspectiva teórica compleja, resulta indispensable esclarecer las decisiones metodológicas para avanzar en el análisis de otras clases; es decir, delimitar las adaptaciones para el contexto mexicano, sus escuelas, sus profesores y nuestras condiciones para la investigación.

En segundo lugar, tanto el proceso metodológico como los resultados muestran que el análisis de clase no puede considerarse como parte de un proceso de evaluación para determinar idoneidad en el cargo. Primero es necesario dimensionar las exigencias que el análisis plantea al profesor: disponibilidad de tiempo (que no necesariamente debe estar fuera de su jornada laboral), recursos técnicos (para grabar y transcribir) y acompañamiento pedagógico cercano para ver su trabajo y tomar conciencia de las decisiones que motivan su actuar. Una lista de tareas evaluativas (INEE, 2018) muy seguramente no es suficiente para concretar un análisis de clase que contribuya a la mejora de la práctica docente. Como tampoco lo son los instrumentos estandarizados como cuestionarios, exámenes y listas de cotejo que han caracterizado las evaluaciones del desempeño docente en México (Martínez, 2016).

En cambio, el proceso metodológico que aquí utilizamos pudiera orientar las prácticas docentes de la profesora en la enseñanza del español, al haberle ayudado a dimensionar los efectos de sus decisiones en las reacciones de los estudiantes. Además, la función de codiseñadores del trabajo prescrito para la clase analizada (y la secuencia didáctica en extenso) nos permitió reflexionar sobre las herramientas didácticas que compartimos con la profesora. Por ejemplo, la gestión de clase no se tomó en cuenta como parte de la preparación para la intervención; mientras que la revisión de los contenidos de enseñanza y el material 
didáctico no fue suficiente para lograr que la profesora se sintiera cómoda al desarrollar la secuencia.

En congruencia con el enfoque didáctico disciplinar de este estudio, no podemos perder de vista la especificidad de los contenidos de enseñanza y de los retos que imponen al profesor; no obstante, esta enseñanza se concreta en el marco de un espacio áulico a cargo de un profesor particular configurado con una fuerte carga histórica que comparte con otros docentes. Como se mencionó al inicio del artículo, las investigaciones etnográficas y didácticas han contribuido al respecto; sin embargo, todavía es necesario vincular sus puntos de vista en la búsqueda de una mejor comprensión del trabajo docente.

Sabemos que es difícil considerar todos los factores que inciden en el trabajo del profesor, de ahí la dificultad de caracterizarlo a la que alude Bronckart (2007), pero no se puede obviar la naturaleza de las actividades de enseñanza y atribuir lo sucedido en clase únicamente al maestro.

\section{Referencias}

Arteaga, P. (2011). Los saberes docentes de profesores en escuelas con grupos multigrado. Consejo Mexicano de Investigación Educativa.

Bronckart, J. (2007). Desarrollo del lenguaje y didáctica de las lenguas. Miño \& Dávila.

Bronckart, J. (2013). En las fronteras del Interaccionismo sociodiscursivo: aspectos lingüísticos, didácticos y psicológicos. En D. Riestra (Comp.), Terceras Jornadas Internacionales de Investigación y Prácticas en Didáctica de las Lenguas y las Literaturas (pp. 39-59). Geise.

Cano, A. (2017). Enseñar y aprender español en una telesecundaria de contexto indígena. Eón.

Civera, A. (20-24 de noviembre de 2017). Miradas a la práctica docente en primaria. Memoria electrónica de XIV Congreso Nacional de Investigación Educativa, San Luis Potosí, México.

https://www.comie.org.mx/congreso/memoriaelectronica/v14/doc/2350.pdf

Clot, Y. y Fäita, D. (2000). Genres et styles en analyse du travail: concepts et méthodes [Géneros y estilos en el análisis del trabajo: conceptos y métodos]. Travailler: Revue Internationale de Psychopathologie et de Psychodynamique du Travail, 4, 7-42.

https://psychanalyse.cnam.fr/medias/fichier/texteclot4 1306851012723.pdf

Dolz, J., Gagnon, R. y Mosquera, S. (2009). La didáctica de las lenguas: una disciplina en proceso de construcción. Didáctica. Lengua y Literatura, 21,117-141.

https://revistas.ucm.es/index.php/DIDA/article/view/DIDA0909110117A

Edwards, V. (1993). La relación de los sujetos con el conocimiento. Revista Colombiana de Educación, (27). https://doi.org/10.17227/01203916.5304

Fernández, G. y Clot, Y. (2010). Entrevistas en auto-confrontación: un método en clínica de la actividad. Informática na Educacao: Teoría \& Prática, 13(1), 11-16.

http://laboreal.up.pt/files/articles/2007 07/es/15 19es.pdf

Goicoechea, M. (2017). Trabajo real y trabajo efectivo. La actividad de trabajo docente y la teoría del diálogo. En D. Riestra (Comp.), Quintas Jornadas Internacionales de Investigación y Prácticas en Didáctica de las Lenguas y las Literaturas (pp. 36-46). UNRN.

Goicoechea, M. V. y Goicoechea, M. A. (2017). Análisis de la actividad registrada en situación de clase: la educación física de nivel primario en contexto. Poiésis-Revista do Programa de Pós-Graduação em Educação, 11(20), 433-450. http://dx.doi.org/10.19177/prppge.v11e202017433-450 
Goicoechea, M., Riestra, D. y Vodnik, V. (2011). Análisis del trabajo docente: las interacciones en las clases de Lengua [CD]. En D. Riestra (Comp.), II Jornadas internacionales de Investigación y Prácticas en Didáctica de las Lenguas y las Literaturas (pp. 374-385). Geise.

Guzmán, F. (2018). La experiencia de la evaluación docente en México: análisis crítico de la imposición del servicio profesional docente. Revista Iberoamericana de Evaluación Educativa, 11(1), 135-158.

https://doi.org/10.15366/riee2018.11.1.008

Instituto Nacional para la Evaluación de la Educación. (2018). Manual para el evaluador. Primaria.

http://www.servicioprofesionaldocente.sep.gob.mx/portal-docente-2014-

2018/2018/manuales/Manual Primaria.pdf

López, A. (2007). Libros de texto y profesionalidad docente. Avances en Supervisión Educativa: Revista de la Asociación de Inspectores de Educación de España, 6, 1-13.

https://avances.adide.org/index.php/ase/article/view/282/244

Martínez, F. (2016). La evaluación de docentes de educación básica. Una revisión de la experiencia internacional. INEE. https://publicaciones.inee.edu.mx/buscadorPub/P1/C/233/P1C233.pdf

Riestra, D. (2008). Las consignas de trabajo en la escuela, un instrumento didáctico. Revista Novedades Educativas, 211, 174-183.

Riestra, D. (2017). Enseñar lengua o enseñar literatura, ¿cuál es el modelo didáctico? En D. Riestra (Comp.), V Jornadas Internacionales de Investigación y Prácticas en Didáctica de las lenguas y las literaturas (pp. 120136). UNRN.

Tapia, S. (2017). Análisis del trabajo docente: relaciones entre lo prescripto y lo realizado en clases de lengua y literatura. Revista de la Escuela de Ciencias de la Educación, 2(12), 87-105.

https://doi.org/10.35305/rece.v2i12.310

Tapia, S. y Goicoechea, M. (2015). La enseñanza de la impersonalidad en la escritura de géneros académicos, Tejuelo, 1(21), 181-193. https://relatec.unex.es/revistas/index.php/tejuelo/article/view/1645 